\title{
Clarifying the relationship between torpor and anthropogenic extinction risk in mammals
}

\author{
E. Hanna \& M. Cardillo \\ Macroevolution \& Macroecology Group, Research School of Biology, Australian National University, Canberra, ACT, Australia
}

\section{Keywords}

daily torpor; IUCN Red List; phylogenetically independent contrasts; predation.

\author{
Correspondence \\ Marcel Cardillo, Macroevolution \& \\ Macroecology Group, Research School of \\ Biology, Australian National University, \\ Building 116, Daley Rd, Canberra, ACT \\ 0200, Australia. \\ Email: marcel.cardillo@anu.edu.au \\ Editor: Andrew Kitchener \\ Received 27 October 2013; revised 13 \\ February 2014; accepted 27 February 2014 \\ doi:10.1111/jzo.12136
}

\begin{abstract}
The ability to undertake torpor has been linked with human-mediated extinction risk in mammals, but whether torpor serves to elevate or decrease extinction risk, and the mechanism by which it does so, remain controversial. We attempt to clarify the torpor - extinction risk association in a phylogenetic comparative analysis of 284 Australian mammal species. We show that the association is strongly mediated by body size. When body mass is included as a covariate, regression models show a negative association between the ability to undertake torpor and current threat status. This association is present in two categories of mammal species likely to be at particular risk from introduced predators (medium-sized species and species listed as threatened by predation in the International Union for Conservation of Nature Red List), but there is no association among species not in these categories. This suggests that torpor reduces vulnerability to predators, perhaps by limiting the amount of time spent foraging. However, the association between torpor and extinction risk is also stronger in smaller species, which are more likely to benefit from a reduced energy budget in Australia's low-productivity and unpredictable environment. We conclude that the ability to undertake torpor is clearly an advantage to mammal species in coping with human impacts, and that this advantage is conferred through a combination of reduced exposure to predators and reduced energy requirements.
\end{abstract}

\section{Introduction}

Torpor is an energy-conserving adaptation to seasonally scarce resources (including both food and water) or unpredictably variable availability of resources (Lyman et al., 1982; Munn, Kern \& McAllan, 2010; Geiser \& Brigham, 2012). In species that internally regulate their body temperature, torpor is characterized by a controlled reduction of body temperature and lowered metabolic rate (Geiser, 2004a). This can occur for hours at a time (e.g. daily torpor), or for much longer periods (e.g. seasonal hibernation). Many mammal species, including placentals, marsupials and monotremes, undertake torpor of one kind or another. Torpor in mammals can be either reactive (occurring as a direct, near immediate response when an individual cannot find enough food or water to sustain nontorpor activity) or predictive (where torpor occurs to conserve energy when energy supplies are likely to be low in the near future; Geiser \& Brigham, 2012). For example, the stripefaced dunnart Sminthopsis macroura goes into torpor even when food is available; this is believed to be a coping mechanism to deal with variable food supplies in their arid, unpredictable environment (Geiser, 2004b; Kortner \& Geiser, 2009).

Torpor may play an important role in contemporary conservation, because a number of studies have linked population declines and species risk of extinction (current threat status) to the ability to undertake torpor. One suggestion is that undertaking torpor makes species more vulnerable to predation, as individuals are easy prey while in a state of torpor, thereby elevating extinction risk (e.g. Armitage, 2004). Conversely, it has also been suggested that undertaking torpor is actually a defence against predation, as individuals spend less time foraging and travelling between nesting and feeding sites. This reduces time spent in the open, where they are more vulnerable to predation, and decreases extinction risk (Geiser \& Kortner, 2010; Stawski \& Geiser, 2010). This association could be especially important given that introduced predators are responsible for declines and extinctions of many mammal species (e.g. Johnson, 2006).

The energetic implications of torpor may also influence contemporary extinction risk. On the one hand, torpor may lessen the risk of extinction by reducing individual energy intake, permitting a species to maintain a viable population in areas of scarce or variable resources (Pavey \& Geiser, 2008; Geiser \& Kortner, 2010). However, torpor also carries energetic costs. Rewarming from the lowered body temperature not only has high energetic costs in itself (Warnecke, Turner \& Geiser, 2008), but also reduces time available for foraging, limiting the amount of energy that animals can gain (Ruf \& Heldmaier, 1992). If the energetic cost of rewarming is minimized through basking in the sun (Warnecke et al., 2008), 
individuals may be more exposed to predators. Undertaking torpor also has other costs, such as reduced immunocompetence (Burton \& Reichman, 1999). Reproduction is slowed through mechanisms such as delayed fetal development (Racey \& Swift, 1981) and limited spermatogenesis (Barnes et al., 1986). Milk production can also be slowed (Wilde, Knight \& Racey, 1999), meaning that young grow slower. Whether the energetic benefits of torpor to individual animals outweigh the costs probably depends on the reliability of resource availability in the particular environments they inhabit.

The mechanisms described above, therefore, make a set of contrasting predictions about the links between torpor and contemporary, human-mediated extinction risk. Torpor may be associated with reduced or increased extinction risk, and it may affect extinction risk via the risk of predation, energetic efficiency or both. In this context, it may not be surprising that previous studies of the relationship between torpor and contemporary extinction risk in mammals have found different outcomes. Some studies have found that mammals that undertake torpor have a reduced threat status, both globally (Geiser \& Turbill, 2009) and in Australian mammals (Geiser \& Kortner, 2010). However, torpor shows strong phylogenetic signal in groups, such as marsupials and bats (Cooper \& Geiser, 2008), so it is important for results of studies such as these, that treat species as independent data points, to be replicated using methods that control for phylogenetic nonindependence (Harvey \& Pagel, 1991). Torpor was found to be unrelated to extinction risk in a study of global mammal species that did control for phylogenetic non-independence (Liow et al., 2009). This study focused on whether 'sleep' (undertaking torpor) or 'hide' (e.g. burrowing) behaviour reduced extinction risk through protection from the environment, and these two behaviours were grouped together in most analyses. Data on whether or not torpor itself is undertaken was therefore not included for the majority of species in this study (Liow et al., 2009).

Furthermore, both torpor and species threat status are correlated with a number of life-history traits in mammals. For example, torpor may be associated with basal metabolic rate and body size (McNab, 1983; Cooper \& Geiser, 2008), and extinction risk is associated with body size and a range of other life-history attributes (Cardillo et al., 2008). Although Liow et al.'s (2009) study included traits such as body size in models with the group of sleep-or-hide mammals, only univariate torpor-risk tests were performed for the species with data on torpor. Analysis of the association between torpor and extinction risk in a multivariate framework may account more completely for possible confounding effects of life history or ecology on the torpor-risk association.

We believe, therefore, that there is a need for further investigation to clarify the association between torpor and contemporary, human-mediated extinction risk, using analyses that (1) distinguish the mechanisms of predation and energetics, and (2) account for phylogenetic non-independence, and lifehistory and ecological covariates. In this study, we use Australian mammals to analyse the association between torpor and extinction risk. Australian mammals provide a powerful case study for comparative analyses of extinction risk, as this group accounts for nearly a third of recent mammal extinctions worldwide (Burbidge \& McKenzie, 1989), and approximately $20 \%$ of the remaining Australian mammals are currently considered threatened with extinction (IUCN, 2012). Additionally, a large proportion ( $>40 \%)$ of Australian mammal species undertake some form of torpor. We first test for associations between torpor and extinction risk for all Australian mammals, both with and without biological covariates. We then attempt to clarify the mechanisms underlying the association, by using the International Union for Conservation of Nature (IUCN) Red List to identify species that have become threatened primarily through habitat loss, and those that have become threatened primarily by introduced predators. We also identify two other subsets of species that are more likely to be threatened by introduced predators: (1) non-volant species, and (2) species whose body mass is in the 'critical weight range' (CWR) of 35-5500 g, which are suggested to lie within the preferred prey size range of introduced cats and foxes (Burbidge \& McKenzie, 1989). Under the predation hypothesis, we would expect an association between torpor and extinction risk to be more apparent for species listed by the IUCN as threatened by predation, for non-volant species, and for CWR species. This association could be positive (if torpor puts species at greater risk from predators) or negative (if torpor protects species from predators). Under the energetic hypothesis, on the other hand, we would expect an association between torpor and extinction risk to be most apparent for species threatened by habitat loss, as their capacity to obtain sufficient resources from the environment to support viable populations may already have been restricted. Again, the association could be positive (if extinction risk is more strongly influenced by the energetic costs of torpor) or negative (if extinction risk is more strongly influenced by the energetic advantages of torpor).

\section{Materials and methods}

The current threat status of all Australian native terrestrial mammals was obtained from the IUCN Red List (IUCN, 2012). Threat status was converted to an ordinal scale from 0 to 5 following Purvis et al. (2000). In addition, we coded extinction as a binary variable (extinct versus extant), to allow direct comparison with Geiser \& Turbill (2009). We recorded data on nesting level from Van Dyck \& Strahan (2008), coded as follows: 1 = below ground (burrows, soil cracks, caves and/or rock fissures); 2 = both below ground and on ground level nests; 3 = ground level; $4=$ both ground level and tree hollows; and $5=$ tree hollows, tree canopy and/or tree branches. Body mass and basal metabolic rate were obtained from Jones et al. (2009). The primary threatening process (or processes) for each threatened species (predation and/or habitat loss) was from the IUCN Red List (IUCN, 2012). Species were not considered threatened by predation if they were listed as threatened by competition or disease transmission by other species without being hunted by another species. A species was considered threatened by habitat loss whether it was listed as either a broad-scale or local event. We included 
'habitat fragmentation' as habitat loss, but we did not include 'habitat degradation', because its definition is more ambiguous and it is difficult to make a valid judgement on how habitat degradation contributes to extinction risk. If there was no information about the threats faced by the species, the species was excluded from these analyses.

Data indicating whether or not a species undergoes torpor was from Geiser \& Kortner (2010) and their references, updated with additional data from a literature search of studies published since 2009 (see Supporting Information Table S1). Data on torpor, coded as a binary variable, were available for 271 of the 284 species in our dataset. We defined torpor as periods of controlled reduction of body temperature with lowered metabolic rate (Geiser, 2004a). We did not differentiate between mammals that undertake long and short torpor, following Geiser \& Turbill (2009) and Geiser \& Kortner (2010). Also, relatively few Australian mammals undertake prolonged torpor (Geiser \& Kortner, 2010), and even in those species where hibernation is undertaken, it is geographically variable (Nicol \& Andersen, 2002; Morrow \& Nicol, 2009).

The phylogenetic signal in torpor was tested using the D statistic (Fritz \& Purvis, 2010), which is appropriate for binary variables. This indicated strong phylogenetic signal, necessitating the use of methods that account for phylogeny. For the phylogenetic comparative analyses, we used a species-level mammal supertree (Bininda-Emonds et al., 2007; Fritz, Bininda-Emonds \& Purvis, 2009), to which we added 34 tips representing extinct species and recently taxonomically redefined species. For 19 of these extinct species, no information was available to guide their placement on the tree, so they were grafted on to the base of their genus. As the phylogeny is ultrametric, branch lengths for the grafted species were assumed to be equal to the distance from the tips of the tree to the nodes at which the species were attached. Supporting Information Table $\mathrm{S} 2$ lists the additional species, along with the references used to guide their placement.

We examined the relationship between torpor, extinction risk and other variables, using multiple regression on phylogenetically independent contrasts using the function 'crunch' in the R library caper (Orme et al., 2012). For direct comparison with Geiser \& Kortner (2010), we also repeated analyses using standard, non-phylogenetic multiple regression across species. All predictors were tested as linear terms, with body mass also being tested as a quadratic variable to allow for the possibility that mammal species of intermediate body mass may be more susceptible to introduced predators (Burbidge \& McKenzie, 1989). Biological variables recorded using continuous scale were log-transformed before modelling (body mass, mass-specific basal metabolic rate and average mass of individuals measured for basal metabolic rate). These analyses were carried out on nine subsets of terrestrial mammal species - all species, non-volant species, bats, CWR non-volant species, non-CWR non-volant species, species threatened by habitat loss, species not threatened by habitat loss, species threatened by predation and species not threatened by predation. Models were regarded as statistically acceptable only if the degrees of freedom were at least 20 , or five times the number of predictors. We fitted alternative sets of predictors for each data subset, and ranked models using Akaike information criterion (AIC) (Akaike, 1974) corrected for small samples (AIC : Hurvich \& Tsai, 1989).

\section{Results}

Of the 284 Australian terrestrial mammals listed in the Red List, data on torpor were available for 271; of these, 120 undertake some form of torpor. When the ability to undertake torpor is displayed on the phylogeny (Fig. 1), it is clear that torpor is strongly phylogenetically clustered. This is confirmed by the strong phylogenetic signal indicated by the $\mathrm{D}$ statistic ( $D=-0.46$, probability that the trait distribution results from a Brownian motion model of trait evolution $=0.97$ ). One implication of the strong clustering of torpor for our analyses is that there are relatively few evolutionary transitions between the states of non-torpor and torpor. This means a majority of the phylogenetically independent contrasts have a value of zero, which limits the statistical power of our analyses, although it should not elevate type 1 statistical error.

In independent-contrast regressions with torpor as the sole predictor of extinction risk, there were no significant associations in any of the nine species subsets (Table 1). In nonphylogenetic cross-species regressions, however, most models revealed a significant negative association (Table 1), indicating that the link between the ability to undertake torpor and a reduced extinction risk is generated by phylogenetic pseudoreplication. When biological covariates were included in the models, significant associations between torpor and extinction risk are revealed in six subsets of species (Table 2). In all of these subsets, torpor shows a negative association with extinction risk, indicating that extinction risk is lower in species with the ability to undertake torpor, once the influence of biological factors is accounted for. Torpor emerges as a significant predictor of extinction risk in two of the species subsets we consider to be particularly vulnerable to introduced predators (CWR species and species listed as threatened by predation in the Red List), but not in species that are not included in these categories (species outside the CWR and species not listed as threatened by predation). In species listed as threatened by predation, nesting level also emerges as a significant predictor of risk, with fossorial species less likely to be threatened than those nesting at or above ground level.

\section{Discussion}

There is uncertainty about the influence of torpor on species' extinction risk, and hence the role torpor might play in contemporary conservation. There is also uncertainty about which mechanism (predation or energetic efficiency) may drive any link between torpor and extinction risk. Our study is the first broad-scale, phylogenetic comparative analysis that attempts to clarify whether torpor is positively or negatively associated with extinction risk, and the mechanism by which it operates. We find that the association between torpor and extinction risk is strongly mediated by body mass: when the effect of body mass is controlled for, the ability to undertake 


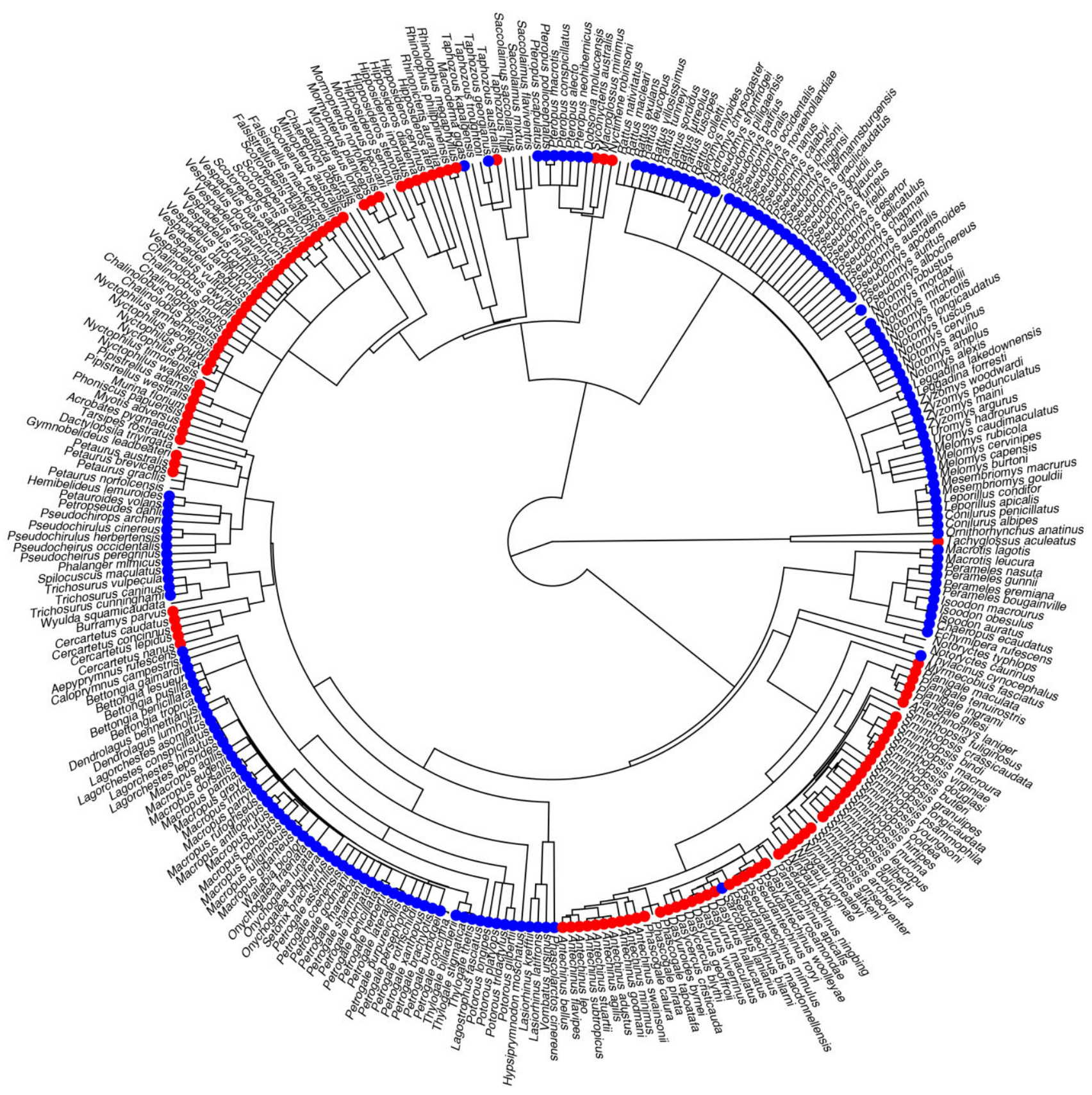

Figure 1 Phylogenetic tree of Australian terrestrial mammals, showing whether torpor is undertaken (red symbols) or not (blue symbols). No symbol equates to no torpor data.

torpor is clearly associated with a reduced risk of extinction. This association is present in the categories of mammal species that we consider particularly vulnerable to predation, but not in species not included in these categories.

The ability to undertake torpor is strongly phylogenetically clustered in Australian mammals. This explains why the significant univariate association between torpor and extinction risk found using non-phylogenetic cross-species regression disappears when we control for phylogeny by using independent contrasts. If torpor is phylogenetically clustered in mammals globally, as it is in Australian mammals, then previous findings of significant univariate relationships between the ability to undertake torpor and lower extinction risk (Geiser \& Turbill, 2009; Geiser \& Kortner, 2010) may at least partially result from phylogenetic non-independence, although the association is consistent with our results. 
Table 1 Relationship between torpor and extinction in Australian mammals using phylogenetically independent contrasts and non-phylogenetic cross-species regression. Values shown are slope estimates, with degrees of freedom in brackets. The response variable is either an ordinally scaled index of extinction risk based on the IUCN Red List, or a binary variable (extinct/extant)

\begin{tabular}{|c|c|c|c|c|}
\hline \multirow[b]{2}{*}{ Subset of species } & \multicolumn{2}{|c|}{ Phylogenetically independent contrasts } & \multicolumn{2}{|c|}{ Cross-species regression } \\
\hline & Ordinal response & Binary response & Ordinal response & Binary response \\
\hline All species & $-0.78(166)$ & $-0.04(166)$ & $-0.85(269)^{\star \star \star}$ & $-0.12(269)^{\star \star *}$ \\
\hline Non-volant & $-1.03(132)$ & $-0.05(132)$ & $-0.73(210)^{* *}$ & $-0.12(210)^{* *}$ \\
\hline Volant & $-0.15(32)$ & $\wedge$ & $-0.66(57)^{*}$ & $-0.10(57)^{*}$ \\
\hline Critical weight range & $0.91(77)$ & $-0.09(77)$ & $-0.65(123)^{\mathrm{a}}$ & $-0.14(123)^{*}$ \\
\hline Non-critical weight range & $-1.51(55)$ & $-0.23(55)$ & $-0.36(73)$ & $-0.05(73)$ \\
\hline Threatened by habitat loss & $-0.09(86)$ & $-0.02(86)$ & $-0.67(126)^{\star}$ & $-0.06(126)^{a}$ \\
\hline Not threatened by habitat loss & $-1.42(93)$ & $-0.06(93)$ & $-0.94(140)^{* * *}$ & $-0.16(140)^{\star * *}$ \\
\hline Threatened by predation & $-0.95(81)$ & $-0.03(81)$ & $-0.88(125)^{\star}$ & $-0.16(125)^{\star}$ \\
\hline Not threatened by predation & $0.02(98)$ & $-0.05(98)$ & $-0.31(141)^{\mathrm{a}}$ & $-0.05(141)^{\mathrm{a}}$ \\
\hline
\end{tabular}

${ }^{\mathrm{a}} 0.05<P \leq 0.1,{ }^{*} 0.01<P \leq 0.05,{ }^{*} 0.001<P \leq 0.01,{ }^{* * *} P \leq 0.001$.

$\wedge$ Not possible to get result from test as there is only one extinct bat.

Table 2 Models of extinction risk using phylogenetically independent contrasts, for six subsets of Australian terrestrial mammal species. Three species subsets for which no significant predictors were found are excluded from the table (bats, species outside critical weight range of 35-5500 $\mathrm{g}$ and species not threatened by predation). The models shown here are those with the lowest $\mathrm{AlC}_{\mathrm{c}}$ values of several alternative models. Values shown are slope estimates

\begin{tabular}{|c|c|c|c|c|c|c|}
\hline & All species & Non-volant & Critical weight range & $\begin{array}{l}\text { Threatened by } \\
\text { habitat loss }\end{array}$ & $\begin{array}{l}\text { Not threatened } \\
\text { by habitat loss }\end{array}$ & $\begin{array}{l}\text { Threatened by } \\
\text { predation }\end{array}$ \\
\hline Torpor & $-18.59 * * *$ & $-25.88^{* * *}$ & $-9.40^{* *}$ & $-9.83 * * *$ & $-6.40 * * *$ & $-19.84 * * *$ \\
\hline Body mass & $-2.45^{* * *}$ & $-2.84^{* * *}$ & $-1.56 * * *$ & $-1.37 * * *$ & $-0.53 * *$ & $-4.03 * * *$ \\
\hline Body mass $\times$ torpor & $2.27 * * *$ & $2.99 * * *$ & $1.42 * *$ & $1.51 * * *$ & $0.69 *$ & \\
\hline Nesting level & & & & & & $1.26^{*}$ \\
\hline d.f. & 152 & 127 & 75 & 78 & 87 & 56 \\
\hline
\end{tabular}

${ }^{*} 0.01<P \leq 0.05,{ }^{*} 0.001<P \leq 0.01,{ }^{* * *} P \leq 0.001$.

d.f., degrees of freedom.

Our results indicate that the effects of torpor and body size on extinction risk are closely linked. When torpor and body size are included in multiple regression models, both have a significant effect on extinction risk. The negative associations between body size and extinction risk in our models are unexpected in the context of previous studies showing that larger mammals tend to have higher extinction risk (e.g. Cardillo, 2003, Cardillo et al., 2005, Cardillo et al., 2006), but the interactive and combined effects of body size and torpor probably explain this. The significant torpor $\times$ body mass interaction terms in many of the models suggest that the ability to undertake torpor is especially important in reducing extinction risk for smaller mammals. This could be an indication of the energetic advantages of undertaking torpor. Smaller mammals have higher massspecific metabolic rates than larger mammals, and suffer greater heat loss per gram of body weight than larger mammals because of their relatively higher surface area, resulting in proportionally higher caloric demands (Withers, 1992; Bradshaw, 2003). Hence, the energetic advantages of torpor are likely to be higher for smaller mammals compared with larger mammals. In an unpredictable environment with a highly variable food supply, this may translate into an association between torpor and population growth rates among smaller mammal species, and thus a reduced extinction risk.

However, our results also provide evidence that torpor is a particular advantage for species likely to be especially vulnerable to predation, as suggested by Geiser \& Kortner (2010) and Stawski \& Geiser (2010). In two of the subsets of species we considered to be more at risk from introduced predators (species within the CWR, and species listed as threatened by predation), we found that torpor is associated negatively with extinction risk. On the other hand, we found no association in species not included in these categories. These results contrast with the view that undertaking torpor increases the chance of being a victim of predation (e.g. Armitage, 2004). Evidently, mammals undertaking daily torpor are able to remain sufficiently well-hidden that any additional risk of predation during periods of torpor is outweighed by the reduced time spent foraging in the open, where exposure to predators is higher (Lima \& Dill, 1990; Geiser \& Kortner, 2010; Stawski \& Geiser, 2010). Alternatively, reduced vulnerability to predation among species that undertake torpor could result from a greater tendency for such species to engage in hiding behaviour more generally (Liow et al., 2009). 
The importance of predation to extinction risk is further supported by the best-fitting model for the subset of species threatened by predation, which showed that reduced extinction risk is associated with large size and more fossorial habits, in addition to the ability to undertake torpor. Because Australia lacks very large mammalian predators, large body size is a clear advantage to potential prey species in reducing the risk of predation (Burbidge \& McKenzie, 1989; Johnson \& Isaac, 2009). Burrowing is also likely to reduce the risk of predation, although some previous work suggests that arboreality is also a defence against introduced non-climbing predators, especially foxes (Johnson \& Isaac, 2009).

The strongly conserved phylogenetic distribution of torpor in Australian mammals would suggest that, although torpor has arisen relatively infrequently, it is advantageous enough in evolutionary time that lineages do not often lose the ability to undertake torpor. Our results demonstrate that the ability of mammal species to undertake torpor is also advantageous to contemporary populations exposed to human impacts. We show that torpor is clearly associated with reduced rather than elevated risk of extinction, but the association between torpor and extinction risk is strongly mediated by body size. Our results suggest that protection from predation is likely to be the primary mechanism that produces this association, although the stronger effects of torpor for smaller species could also have an explanation based on energetic efficiency. We conclude that daily torpor confers advantages to smaller mammal species for reasons of both predator protection and energetic efficiency. What remains little understood is the extent to which these associations are peculiar to the highly unpredictable environments, with strongly variable food supplies, that characterize much of Australia, or to environments in which introduced predators exert a strong top-down control on mammal populations. It is possible that these issues could be resolved by comparisons of the associations between torpor and extinction risk among faunas in parts of the world that differ in levels of primary productivity or available energy, and the stability of resource supplies.

\section{References}

Akaike, H. (1974). A new look at the statistical model identification. IEEE Trans. Automat. Contr. 19, 716-723.

Armitage, K.B. (2004). Badger predation on yellow-bellied marmots. The Am. Midl. Nat. 151, 378-387.

Barnes, B., Kretzmann, M., Licht, P. \& Zucker, I. (1986). The influence of hibernation on testis growth and spermatogenesis in the golden-mantled ground squirrel, Spermophilus lateralis. Biol. Reprod. 35, 1289-1297.

Bininda-Emonds, O.R.P., Cardillo, M., Jones, K.E., MacPhee, R.D.E., Beck, R.M.D., Grenyer, R., Price, S.A., Vos, R.A., Gittleman, J.L. \& Purvis, A. (2007). The delayed rise of present-day mammals. Nature 446, 507-512.

Bradshaw, D. (2003). Vertebrate ecophysiology: an introduction to its principles and applications. Cambridge: Cambridge University Press.
Burbidge, A.A. \& McKenzie, N.L. (1989). Patterns in the modern decline of Western Australia's vertebrate fauna: causes and conservation implications. Biol. Conserv. 50, 143-198.

Burton, R.S. \& Reichman, O. (1999). Does immune challenge affect torpor duration? Funct. Ecol. 13, 232-237.

Cardillo, M. (2003). Biological determinants of extinction risk: why are smaller species less vulnerable? Anim. Conserv. 6, 63-69.

Cardillo, M., Mace, G.M., Jones, K.E., Bielby, J., Bininda-Emonds, O.R.P., Sechrest, W., Orme, C.D.L. \& Purvis, A. (2005). Multiple causes of high extinction risk in large mammal species. Science 309, 1239-1241.

Cardillo, M., Mace, G.M., Gittleman, J.L. \& Purvis, A. (2006). Latent extinction risk and the future battlegrounds of mammal conservation. Proc. Natl Acad. Sci. U.S.A. 103, 4157-4161.

Cardillo, M., Mace, G.M., Gittleman, J.L., Jones, K.E., Bielby, J. \& Purvis, A. (2008). The predictability of extinction: biological and external correlates of decline in mammals. Proc. Biol. Sci. 275, 1441-1448.

Cooper, C.E. \& Geiser, F. (2008). The 'minimal boundary curve for endothermy' as a predictor of heterothermy in mammals and birds: a review. J. Comp. Physiol. B. 178, $1-8$.

Fritz, S.A. \& Purvis, A. (2010). Selectivity in mammalian extinction risk and threat types: a new measure of phylogenetic signal strength in binary traits. Conserv. Biol. 24, 1042-1051.

Fritz, S.A., Bininda-Emonds, O.R.P. \& Purvis, A. (2009). Geographical variation in predictors of mammalian extinction risk: big is bad, but only in the tropics. Ecol. Lett. 12, 538-549.

Geiser, F. (2004a). Metabolic rate and body temperature reduction during hibernation and daily torpor. Annu. Rev. Physiol. 66, 239-274.

Geiser, F. (2004b). The role of torpor in the life of Australian arid zone mammals. Aust. Mammal 26, 125-134.

Geiser, F. \& Brigham, R.M. (2012). The other functions of Torpor. In Living in a seasonal world: 123-146. Ruf, T., Bieber, C., Arnold, W. \& Millesi, E. (Eds). Berlin: Springer. Geiser, F. \& Kortner, G. (2010). Hibernation and daily torpor in Australian mammals. Aust. Zool. 35, 204-215.

Geiser, F. \& Turbill, C. (2009). Hibernation and daily torpor minimize mammalian extinctions. Naturwissenschaften 96, 1235-1240.

Harvey, P.H. \& Pagel, M. (1991). Comparative method in evolutionary biology. Oxford: Oxford University Press.

Hurvich, C.M. \& Tsai, C.L. (1989). Regression and time series model selection in small samples. Biometrika 76, 297307.

IUCN (2012) IUCN Red List of Threatened Species [online database]. Version 2012.2. International Union for Conservation of Nature and Natural Resources, IUCN Species Programme, IUCN Red List Unit Cambridge UK. 
Johnson, C. (2006). Australia's mammal extinctions: a 50000 year history. Cambridge: Cambridge University Press.

Johnson, C.N. \& Isaac, J.L. (2009). Body mass and extinction risk in Australian marsupials: the 'critical weight range' revisited. Austral Ecol. 34, 35-40.

Jones, K., Bielby, J., Cardillo, M., Fritz, S., O’Dell, J., Orme, C., Safi, K., Sechrest, W., Boakes, E. \& Carbone, C. (2009). PanTHERIA: a species-level database of life history, ecology, and geography of extant and recently extinct mammals. Ecology 90, 2648.

Kortner, G. \& Geiser, F. (2009). The key to winter survival: daily torpor in a small arid-zone marsupial. Naturwissenschaften 96, 525-530.

Lima, S.L. \& Dill, L.M. (1990). Behavioral decisions made under the risk of predation: a review and prospectus. Can. J. Zool. 68, 619-640.

Liow, L.H., Fortelius, M., Lintulaakso, K., Mannila, H. \& Stenseth, N.C. (2009). Lower extinction risk in sleep-orhide mammals. Am. Nat. 173, 264-272.

Lyman, C.P., Willis, J.S., Malan, A. \& Wang, L.C.H. (1982). Hibernation and torpor in mammals and birds. New York: Academic Press.

McNab, B.K. (1983). Energetics, body size, and the limits to endothermy. J. Zool. 199, 1-29.

Morrow, G. \& Nicol, S.C. (2009). Cool sex? Hibernation and reproduction overlap in the echidna. PLoS ONE 4, e6070.

Munn, A.J., Kern, P. \& McAllan, B.M. (2010). Coping with chaos: unpredictable food supplies intensify torpor use in an arid-zone marsupial, the fat-tailed dunnart (Sminthopsis crassicaudata). Naturwissenschaften 97, 601-605.

Nicol, S. \& Andersen, N.A. (2002). The timing of hibernation in Tasmanian echidnas: why do they do it when they do? Comp. Biochem. Physiol. B Biochem Mol. Biol. 131, 603611.

Orme, C., Freckleton, R., Thomas, G., Petzoldt, T., Fritz, S., Isaac, N. \& Pearse, W., (2012) Caper: comparative analyses of phylogenetics and evolution in R. In: R package version 0.5 .
Pavey, C.R. \& Geiser, F. (2008). Basking and diurnal foraging in the dasyurid marsupial Pseudantechinus macdonnellensis. Aust. J. Zool. 56, 129-135.

Purvis, A., Gittleman, J.L., Cowlishaw, G. \& Mace, G.M. (2000). Predicting extinction risk in declining species. Proc. Biol. Sci. 267, 1947-1952.

Racey, P. \& Swift, S.M. (1981). Variations in gestation length in a colony of pipistrelle bats (Pipistrellus pipistrellus) from year to year. J. Reprod. Fertil. 61, 123-129.

Ruf, T. \& Heldmaier, G. (1992). The impact of daily torpor on energy requirements in the Djungarian hamster, Phodopus sungorus. Physiol. Zool. 65, 994-1010.

Stawski, C. \& Geiser, F. (2010). Fat and fed: frequent use of summer torpor in a subtropical bat. Naturwissenschaften 97, 29-35.

Van Dyck, S. \& Strahan, R. (2008). The mammals of Australia. 3rd edn: Sydney: New Holland Publishers.

Warnecke, L., Turner, J.M. \& Geiser, F. (2008). Torpor and basking in a small arid zone marsupial. Naturwissenschaften 95, 73-78.

Wilde, C.J., Knight, C.H. \& Racey, P.A. (1999). Influence of torpor on milk protein composition and secretion in lactating bats. J. Exp. Zool. 284, 35-41.

Withers, P.C. (1992). Comparative animal physiology. Fort Worth: Saunders College Publishing.

\section{Supporting information}

Additional Supporting Information may be found in the online version of this article at the publisher's web-site:

Table S1. Database of extinction risk, torpor and biological traits of Australian mammal species.

Table S2. Details of species added to the published mammal phylogeny. 